\title{
An update of menopausal hormone therapy: present perspective
}

Volume 9 Issue 2 - 2018

\section{Introduction}

Menopause is a physiological event which is diagnosed after one year of cessation of menstruation and leads to cardiovascular consequences, osteoporosis and dementia as long term consequences. ${ }^{1-3}$ Menopausal Hormone Therapy (MHT) is theoretically the mainstay for managing the acute symptoms of estrogen deficiency e.g. vasomotor instability, psychosomatic symptoms, genito-urinary syndrome as well as for the prevention of long term consequences of estrogen deprivation. Still MHT is a complex issue to be addressed as constantly changing reports and recommendations regarding MHT have been challenging practitioners. Elderly population of the world is on rise and also life expectancy in women so women are expected to live almost one third of their life postmenopausally. Menopause before the age of 40 is considered as premature menopause. In India the average age of menopause is 47.3 years. ${ }^{4}$ Clear understanding of menopause management is therefore necessary to deal with this group of women.

The field of menopause management has seen many ups and downs since the beginning of estrogen therapy in 1940s. Initially multiple studies reported its beneficial effects for prevention of osteoporosis, cardiovascular events and psychosomatic symptoms so use of MHT was considered the most effective solution. ${ }^{5}$ It's use was increased to nearly double from 1960 to 1975 as unopposed estrogen therapy to all menopausal woman with or without uterus. This trend changed with the appearance of endometrial cancers and registered a decline in usage. Evidenced by further studies and use of progestins as protective agent the use of estrogen-progesterone combined/alone therapy again gained popularity. Later in 2002 preliminary results from WHI ${ }^{6-9}$ caused a major setback and preventive role of MHT again gone into disrepute. ${ }^{10}$ The WHI results conclusively demonstrated the link between the most commonly used combined hormone preparation of estrogen plus progestin and adverse outcomes such as breast cancer and cardiovascular disease. Million Women Study report assessing breast cancer incidence in Belgium between 2007 and 2008 further showed a significant drop in breast cancer incidence attributed to the decrease in HRT use. ${ }^{11,12}$ After more than a decade of fear and confusion regarding MHT, reanalysis of the Women's Health Initiative data and the results of recent studies have provided some clarity regarding the balance of risks and benefits of systemic MHT.

The current research advocates the benefits from MHT when initiated within 10 years of menopause in women with appropriate indications with continuation thereafter for an intermediate period for the prevention of chronic disease. Age and years since menopause are now known to be important variables affecting the benefit-risk profile.

For symptomatic menopausal women who are under 60 years of age or within 10 years of menopause, the benefits of MHT generally outweigh the risks. ${ }^{13}$

Updated guidelines for MHT use have been provided by the Indian Menopause Society, North American Menopause Society, International Menopause Society, British Menopause Society,

\section{Seema Sharma}

Department of Obstetrics \& Gynecology, Mahatma Gandhi Medical College \& Hospital, India

Correspondence: Dr. Seema Sharma, Professor, Department of Obstetrics \& Gynecology, Mahatma Gandhi Medical College \& Hospital (MGMCH), Jaipur, India, Tel 09829I 63290, Email drseemadsharma@gmail.com

Received: November 02, 2017 | Published: March 27, 2018

and many others, with consensus statements incorporating this concept. Hormone therapy is approved by FDA for four indications: bothersome vasomotor symptoms, prevention of bone loss, hypoestrogenism caused by hypogonadism, castration, or premature ovarian insufficiency and genitourinary symptoms. ${ }^{2}$

Decision of MHT should therefore be taken after explaining HT risk to the patients in terms of odds ratio, followed by the decision of type of preparation to be used. Meta-analysis of FDA-approved estrogen trials found no evidence of a significant difference in effectiveness between estradiol and conjugated equine estrogen in treating vasomotor symptoms \& progestins should be used for endometrial protection in woman with intact uterus.

According to International Menopause Society guidelines women experiencing a spontaneous or iatrogenic menopause before age 45 and particularly before age 40 are at higher risk of cardiovascular disease and osteoporosis. In these women, in the absence of contraindications, MHT is advised at least until the average age of menopause. MHT should not be recommended without a clear indication for its use. Women taking MHT should have at least an annual medical consultation. Dose and duration of MHT should be consistent with treatment goals. Decision to continue MHT should be at the discretion of the well-informed woman and her health professional. Consideration of MHT should be part of an overall strategy including lifestyle recommendations regarding diet, exercise, smoking cessation and safe levels of alcohol consumption for maintaining the health of peri- and postmenopausal women and MHT must be individualized and tailored according to symptoms, the need for prevention, personal and family history, results of investigations and each woman's preferences and expectations. ${ }^{14}$

A latest Cochrane review included 22 studies on long term hormone therapy concluded that HT may be unsuitable for some women, including those at increased risk of cardiovascular disease, increased risk of thromboembolic disease i.e. those with obesity or a 
history of venous thrombosis or increased risk of some types of cancer e.g. breast cancer in women with a uterus.

HT is neither indicated for primary or secondary prevention of cardiovascular disease or dementia, nor for prevention of deterioration of cognitive function in postmenopausal women. Although HT is considered effective for the prevention of postmenopausal osteoporosis, it is generally recommended as an option only for women at significant risk for whom non-oestrogen therapies are unsuitable. Data are insufficient for assessment of the risk of long-term HT use in perimenopausal women and in postmenopausal women younger than 50 years of age. ${ }^{15}$

\section{Conclusion}

The use of estrogen as hormone therapy is like a double edged sword that relieves menopausal symptoms at one end but pose serious health risks including cardiovascular morbidity and cancers at the other end. Therefore, care providers should counsel the postmenopausal women who are going to use MHT to determine whether the benefits outweigh the risks in their particular case.

MHT should be used at the lowest dosages and for the shortest duration necessary to achieve symptomatic relief. Dosages and durations may vary greatly among individual patients. Low-dose regimens may lower the risk of serious adverse effects treatment must always be individualized and constantly reviewed.

\section{Acknowledgements}

None.

\section{Conflicts of interest}

Authors declare there is no conflict of interest in publishing the article.

\section{References}

1. Shagaf $\mathrm{H}$ Bakour, Jennifer Williamson. Latest evidence on using hormone replacement therapy in the menopause. The Obstetrician \& Gynaecologist. 2015;17:20-8.

2. The NAMS 2017 Hormone Therapy Position Statement Advisory Panel. The 2017 hormone therapy position statement of The North American Menopause Society. Menopause. 2017;24(7):728-753
3. Jyotsna VP. Postmenopausal hormonal therapy: Current status. Indian J Endocrinol Metab. 2013;17(Suppl 1):S45-S49.

4. Meeta, Digumarti L, Agarwal N, et al. Clinical Practice Guidelines on Menopause: An executive summary and recommendations. J Midlife Health. 2013;4(2):77-106.

5. Prentice RL, Manson JE, Langer RD, et al. Benefits and Risks of Postmenopausal Hormone Therapy When It Is Initiated Soon After Menopause. Am J Epidemiol. 2009;170(1):12-23.

6. Nelson HD, Walker M, Zakher B, et al. Menopausal hormone therapy for the primary prevention of chronic conditions: A systematic review to update the U.S. Preventive Services Task Force recommendations. Ann Intern Med. 2012;157 (2):104-113.

7. Women's Health Initiatives. Lifespan Health Services Unit Division of Public Health Nebraska Department of Health and Human Services. Annual Report-2015-16.

8. Women's Health Initiative. Long Life Study.

9. Women's Health Initiative. Participants - Home.

10. Antoine C, Ameye L, Paesmans M, et al. Update of the evolution of breast cancer incidence in relation to hormone replacement therapy use in Belgium. Maturitas. 2012;72(4):317-323.

11. Banks E, Canfell K. Invited Commentary: Hormone therapy risks and benefits--The Women's Health Initiative findings and the postmenopausal estrogen timing hypothesis. Am J Epidemiol. 2009;170(1):24-8.

12. Rossouw JE, Manson JE, Kaunitz AM, et al. Lessons learned from the Women's Health Initiative trials of menopausal hormone therapy. Obstet Gynecol. 2013;121(1):172-6.

13. Chew F, Wu X. Sources of information influencing the state-ofthe-science gap in hormone replacement therapy usage. PLoS One. 2017;12(2):e0171189.

14. Baber RJ, Panay N, Fenton A. 2016 IMS Recommendations on women's midlife health and menopause hormone therapy. Climacteric. 2016;19(2):109-50.

15. Marjoribanks J, Farquhar C, Roberts H, et al. Long-term hormone therapy for perimenopausal and postmenopausal women. Cochrane Database Syst Rev. 2012;(7):CD004143. 